\title{
Research of Image Edge Detection Based on Mathematical Morphology
}

\author{
Xiufang Wang ${ }^{1}$, Xingyuan Zhang ${ }^{1}$ and Running $\mathrm{Gao}^{2}$ \\ 1. Northeast Petroleum University School of Electrical Engineering \& Information, \\ Daqing, China \\ 2. 180 w. big springs rd. apt.23, Riverside, California, PC: 92507 \\ wxfdqpi@163.com,wind00sound@126.com
}

\begin{abstract}
During the image edge detection with mathematical morphology, using single form and single scale of structural element will lose the information of other forms of elements. And the ability of anti-noise is weak. An adaptive algorithm for image edge detection based on multistructural and multi-scale is proposed in this paper. For single form of structural element, we add weight coefficients on each result of different scale elements according to the inverse ratio of information entropy. For single scale of structural element, we add weight coefficients on each result of different form elements according to the direct ratio of information entropy. At last, we construct a series-parallel form of edge detector according to the algorithm process and get the final edge image after the fusion of edge images. The experiment shows that the improved algorithm not only can retain rich edge information but also has good performance to remove the noise.
\end{abstract}

Keywords: edge detection, mathematical morphology, anti-noise, multi-scale, multi-form

\section{Introduction}

The edge of image is one of the basic characteristics of image and edge detection is very important in image processing. In gray image, image edge reflects the discontinuity of gray and contains important information of image. Many applications which include feature extraction, segmentation and match of image need to use the edge information of image. There are many algorithms for image edge detection, for example, the classical edge detection algorithms like Roberts, Sobel and Prewitt [1-3]. The computational speed of these operators is faster, but ability of anti-noise is weak and the edge details are not complete. The Canny operator based on optimization algorithm was proposed in 1986 [4], it makes anti-noise performance and precision reach a certain balance. The disadvantage is the large amount of calculation, so the efficiency of computation is low and performance of real-time is poor. In recent years, there are some popular algorithms such as Neural Networks, Fractal Theory and Wavelet are also used in the field of edge detection but the disadvantage is that the generality and the ability to suppress noise are weak.

Mathematical morphology is a new nonlinear image processing technique and the focus is geometric relationship of image [5]. Mathematical morphology is based on strict mathematical theory and geometry. The algorithm is simple and it has the advantage of parallel processing so the speed of operation is fast. The geometric attribute of image geometric attributes is the focal point of the algorithm [6]. Due to different shapes of structure elements have different attributes of nature, so the algorithm can maintain the rich natural features of image by determining the 
relationship between each part of image according to them [7]. At the same time, for different forms of noises, the diversity of structural elements can also guarantee the edge detection algorithm has corresponding anti-noise operator for each kind of noise [8].

But single form and single scale structure element have limitations in the complex image edge detection. They are easy to lose the information of the other geometry. And to the image which has different strength of noise, the ability of anti-noise is weak. Aiming at this problem, many scholars have published a lot of research achievements. An edge detection algorithm based on multi-form structure elements is proposed in literature [9].The advantages of this algorithm is that it has a better performance on edge detection to the image which has several kinds of noises. An edge detection algorithm based on multiscale structural elements structure elements is proposed in literature [10].The advantages of this algorithm is that it has a better performance on edge detection to the image which has several kinds intensities of noise. Although the edge detection algorithms above increase the ability of anti-noise to a certain extent, they didn't consider for a single form element, the information entropy is different for the detection of each kind of scale, so the ability for filtering noise and retention of edge details is not same. Small scale structure elements can filter most of the noise information, but the information entropy is small; more edge details can be maintained by large scale structure element, but it contains a lot of noise information, so the information entropy is big. In this paper, for multi-form structure elements, we use direct ratio of information entropy to determine weight coefficient. And for multi-scale structural elements, we use inverse ratio of information entropy to determine weight coefficient. Then we fuse the edge detection of images by series-parallel edge detector which is designed in this paper. Then we get the final image which is the fusion of different scales and structures of edge detection information [11]. The simulation results show that the improved algorithm not only can better keep the edge information, but also has good performance of anti-noise.

The rest of this paper is organized as follows: The second part is the modeling of morphological edge detection in gray-scale. The principle and calculation formula of the improved algorithm is elaborated in the third part. The fourth part is the simulation and performance evaluation of the improved algorithm. The fifth chapter is the summary of the paper.

\section{The Modeling of Mathematical Morphology in Edge Detection}

Initially, mathematical morphology is used for binary image, and gray-scale morphology is an extension of the binary morphology on the gray space [12]. In traditional edge detection of gray-scale morphology [13], $f(x, y)$ is supposed as function of target image; $b(x, y)$ is the operator of structure element; $I_{d}(x, y)$ is the expansion operator; $I_{e}(x, y)$ is the corrosion operator; $G(x, y)$ is the gradient operator. The expansion operator of edge detection which uses single form and single scale structural element is:

$$
I_{d}(x, y)=f(x, y) \oplus b(x, y)-f(x, y)
$$

The corrosion operator of edge detection is:

$$
I_{e}(x, y)=f(x, y)-f(x, y) \Theta b(x, y)
$$


The operator of morphological gradient is:

$$
G(x, y)=I_{d}(x, y)-I_{e}(x, y)
$$

The improved algorithm is proposed in literature [14], it can remain the edge details and reduce the ambiguity of edge but the result may be added some noise information after the fusion. The algorithm is improved again in literature [15]. A multi-form, multi-scale edge detection algorithm is designed and adaptive weighting coefficients are added by using information entropy. But the edge details of result are too rich and the ability of suppressing noise is poor by using large scale structure elements. The property of small scale structural element is opposite. So the algorithm dose not choice the appropriate coefficient. The use of multi-form structure elements extracts more natural information of the image, but the ability to restrain noise didn't get significant improvement. The algorithm of this paper is an improved algorithm based on literature [15], so the following calculation formulas in literature [15] are listed as follows:

$$
\begin{gathered}
I_{\min }(x, y)=\min \left[I_{d}(x, y), I_{e}(x, y), G(x, y)\right] \\
I_{\max }(x, y)=\max \left[I_{d}(x, y), I_{e}(x, y), G(x, y)\right] \\
I_{d e c}(x, y)=I_{\max }(x, y)-I_{\text {min }}(x, y)
\end{gathered}
$$

The edge detection operator is:

$$
I(x, y)=I_{d}(x, y)+0.5 I_{d e c}(x, y)
$$

\section{The Improved Algorithm of Edge Detection}

\subsection{Theory of the Improved Algorithm}

According to the introduction of mathematical morphology and several improved algorithms above, the improved algorithm will be elaborated in detail in this section. Due to the information entropy for elements which have same form and different scale are different and the ability of anti-noising is not same too. First of all, for single form of element: if $b_{i j}$ is the information entropy of edge image for each scale element; $B_{i j}$ is the weight coefficient of each scale element, the subscript $i$ is the serial number of multi-form element, the subscript $\mathrm{j}$ is the serial number of multi-scale element and the subscript $n$ is the amount of multi-form elements. We use inverse ratio of information entropy to determine the numerical value of $B_{i j}$. The calculation formula is as follows:

$$
B_{i j}=\frac{\frac{1}{b_{i j}}}{\frac{1}{b_{i 1}}+\frac{1}{b_{i 2}}+\cdots+\frac{1}{b_{i n}}}
$$

And then we fuse the several edge detections of each scale which belong to single form element to get the single form serial test results. For other forms of structural elements, the calculation is in accordance with the method above. And then the weight coefficient $A_{i}$ for each signal form is calculated according to the direct ratio of information entropy. If $a_{i}$ is the sum of information entropy for each single form 
structure element, the subscript $\mathrm{i}$ is the serial number of multi-form element and the subscript $m$ is the amount of multi-form elements.Then the calculation formula of $A_{i}$ is:

$$
A_{i}=\frac{a_{i}}{a_{1}+a_{2}+\cdots+a_{m}}
$$

If $I_{i}$ is the corresponding information entropy of image edge, $p(i)$ is the probability for pixel which the gray value is $i$, then the numerical value of $a_{i}$ and $b_{i j}$ can be calculated according to the formula as follows:

$$
I_{i}=-\sum_{i=0}^{255} p(i) \times \log _{2} p(i)
$$

If the amount of multi-scale elements is $\mathrm{M}$, the amount of multi-form element is $\mathrm{N}$. After the calculation of the edge image $I(x, y)$ for each element, the weight coefficient of single scale element $b_{i j}$ and the weight coefficient of single form element $A_{i}$, the final edge image can be calculated according to the final formula as follows:

$$
I(x, y)=\sum_{i=1}^{N} A_{i}\left(\sum_{j=1}^{M} B_{i j} \times I_{i j}(x, y)\right)
$$

Through the description of the improved algorithm, a series-parallel edge detector is built in this paper. For each serial sequence, the terminal result is the fusion of image with each form. For each parallel sequence, the terminal is multiplied with the weight coefficient $A_{i}$. And the final image is the sum of all the parallel sequence. If the amount of multi-scale elements is $\mathrm{m}$, the amount of multi-form element is $\mathrm{n}$. The series-parallel form of edge detector is shown in Figure 1:

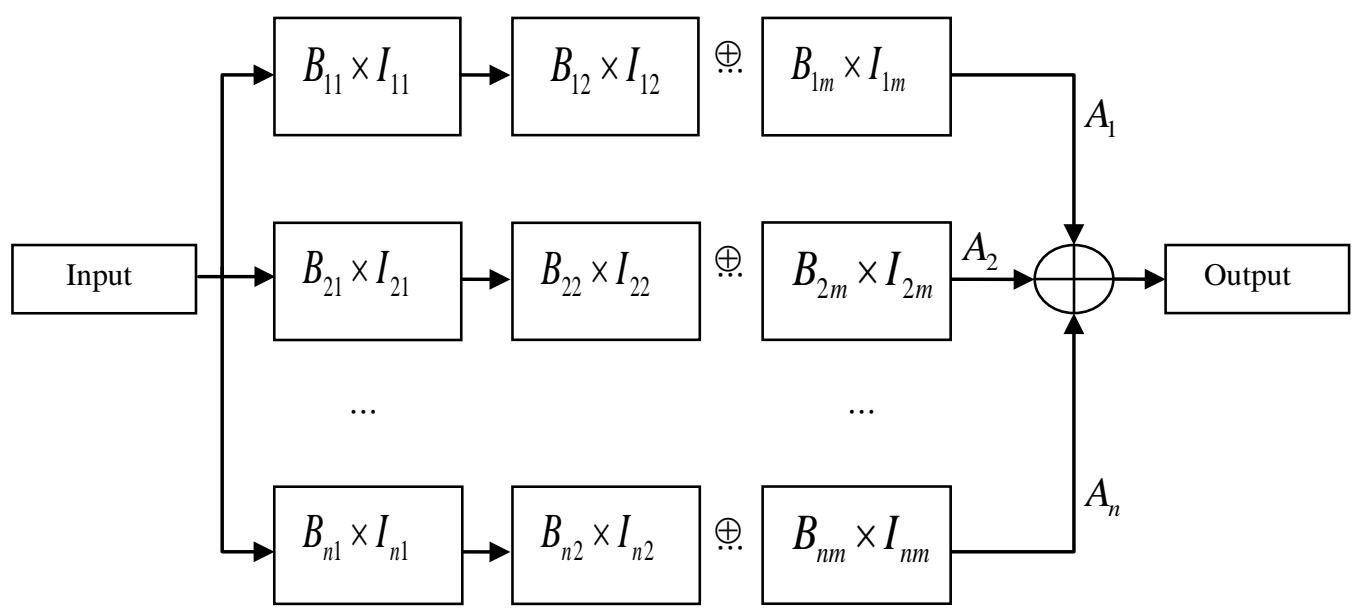

Figure 1. Series-parallel Edge Detector 


\subsection{The Description of the Algorithm}

1. The edge image of the original image for single shape structure and single scale element can be calculated by formula (7).

2. The information entropy of all the edge images is calculated according to formula (10).

3. The weight coefficient of each scale structure is calculated according to formula (8).

4. The fusion of edge image for single form structure is gotten according to the series edge detector. Then we calculate the information entropy. For other forms of structures, repeat steps from (1) to (4).

5. The weight coefficients for different forms of structure elements are calculated by formula (9) and we use the parallel edge detector to get the final edge detection image with formula (11).

\section{The Results and Analysis of Simulation}

We choose MATLAB7.1 to be the experiment environment of this algorithm and select figure of camera man as the test image. First of all, we compare improved algorithm with two kinds of classical edge detection operators Sobel and Canny to verify that the improved algorithm can retain more details. The results of simulation are as follows:

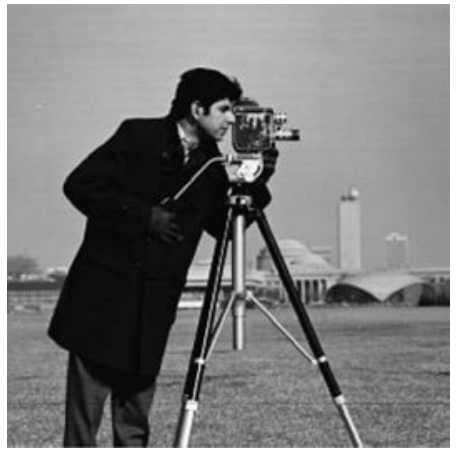

a. Original Picture

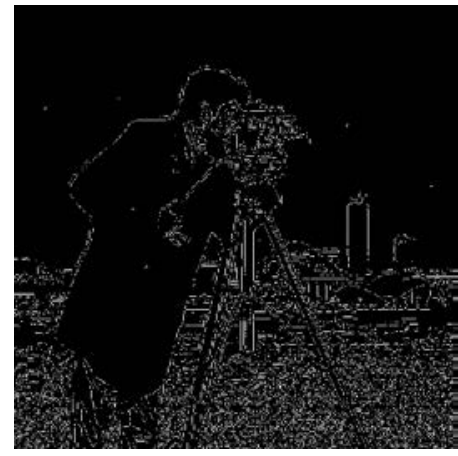

b. Canny Operator



d. Improved Operator

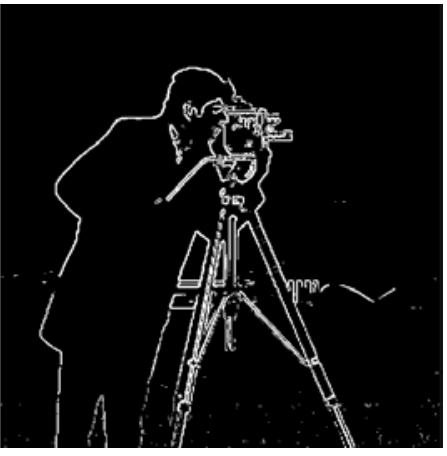

c. Sobel Operator

Figure 2. The Image Edge Detection Results of Non-pollution

According to the analysis of simulation results we can see that Canny operator can detect more edge details but there is too much details of the edge, so the edge information is confused. Sobel operator can detect the edge of subject information very well, but it will lose 
a lot of edge details. Although the edge of the image is thin according to the algorithm in this paper, most of the edge information which is weak can be detected.

Secondly, in order to verify the improved algorithm of this paper has performance improvement in suppress noise, we choose three scenes. The first one is the scene in which the picture is added salt and pepper noise with density of 0.01 . The image edge detection result is shown in Figure 3. Through the analysis of simulation results we can see that Canny operator will also include noise into the edge information. And for Sobel the capability of noise suppression is weak. The algorithm in literature [15] has certain ability of inhibition for noise, but the edge will become blurred. The improved algorithm in this paper can filter most of the noise and retain the information of edge.

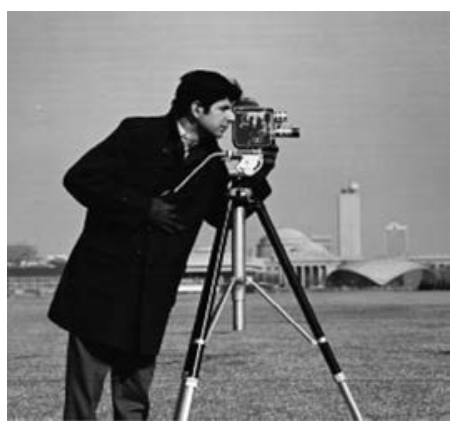

a. Original Picture

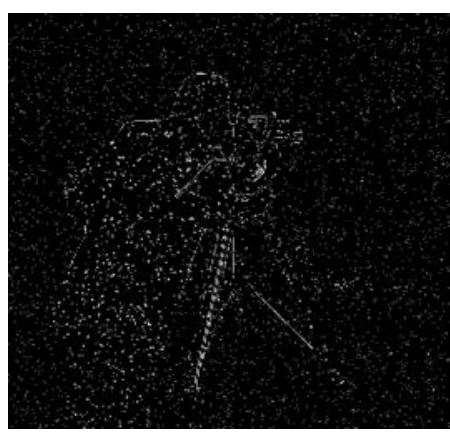

d. Sobel Operator



b. Salt and Pepper Image

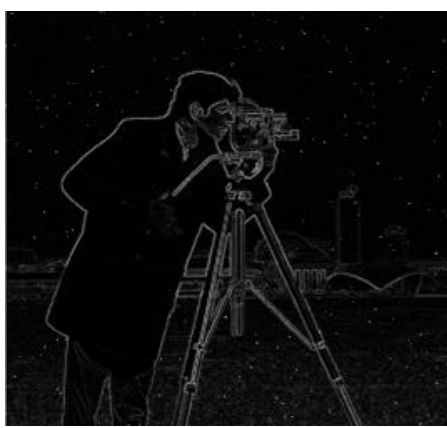

e. Algorithm in Literature [15]

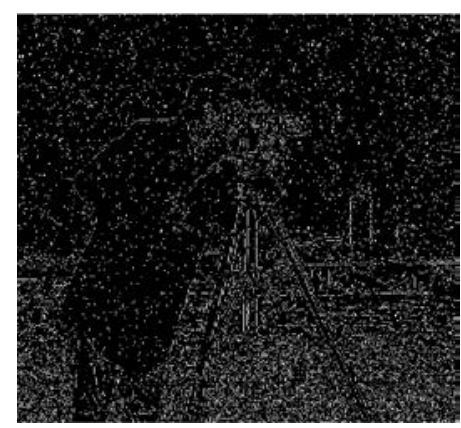

c. Canny Operator



f. Improved Operator

Figure 3. The Edge Detection Results of Salt and Pepper Image

In the second scene, we add salt and pepper noise whose density is 0.03 in the picture, the simulation results are shown in Figure 4:

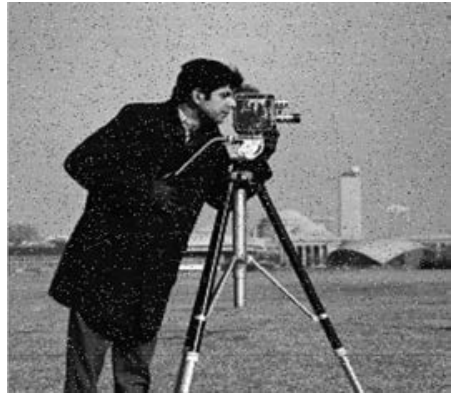

a. Original Picture

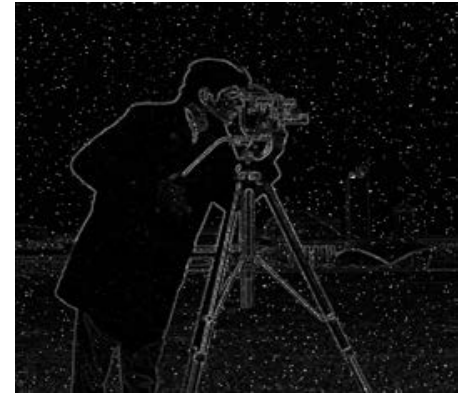

b. Algorithm in Literature [15]



c. Improved Operator

Figure 4. The Edge Detection Results of Salt and Pepper Image 
In the third scene, we add salt and pepper noise which the density is 0.03 to the picture. The simulation results are shown in Figure 5:

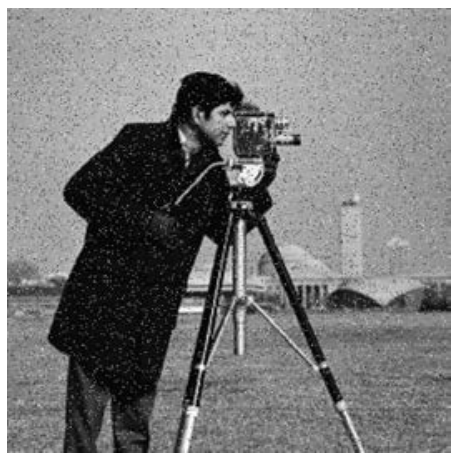

a. Original Picture



e. Algorithm in Literature [15]

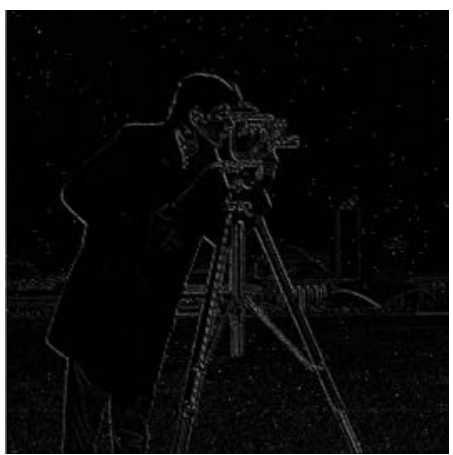

f. Improved Operator

Figure 5. The Edge Detection Results of Salt and Pepper Image

According to the results of algorithm in literature [15] we can see that with the density of noise increasing gradually, the edge information is seriously affected by noise and the edge image is more and more dim. In the case of that the noise is serious, the improved algorithm in this paper will make the edge image appear breakage, the edge image is still clear and most of the noise is filtered.

Table 1 is a quality evaluation index of PSNR (Peak Signal to Noise Ratio) and SNR (Signal to Noise Ratio) in order to evaluate the anti-noise performance of the algorithm in literature [15] and the improved algorithm in this paper. Signal-to-noise ratio is the ratio of signal and noise. Peak signal-to-noise ratio is according to the ratio of the max power of signal and the power of noise.

In the experiments, we join in the salt and pepper noise with density of $0.01,0.03,0.05$ respectively, the results of PSNR and SNR are as follows:

Table 1. Statistical Parameters Contrast of the Salt and Pepper Noise Image

\begin{tabular}{|c|c|c|c|c|}
\hline $\begin{array}{c}\text { The } \\
\text { Density } \\
\text { of Noise }\end{array}$ & \multicolumn{2}{|c|}{$\begin{array}{c}\text { The Algorithms of } \\
\text { Literature [15] }\end{array}$} & \multicolumn{2}{|c|}{ Improved Algorithm } \\
\cline { 2 - 5 } & PSNR(db) & SNR(db) & PSNR(db) & SNR(db) \\
\hline 0.01 & 28.8927 & 7.5898 & 32.7345 & 11.4208 \\
\hline 0.03 & 27.0592 & 5.7992 & 29.6259 & 8.3579 \\
\hline 0.05 & 25.4153 & 4.3978 & 27.947 & 6.9441 \\
\hline
\end{tabular}

In the case of noise with the three densities, the SNR and PSNR of improved algorithm in this paper are greater than the algorithm in literature [15].That is to say, to evaluate from the data, the improved algorithm enhances the ability of anti-noise greatly. 


\section{Conclusion}

In this paper, the general algorithm of mathematical morphology edge detection is improved. An algorithm of edge detection based on multi-scale and multi-form is proposed. The purpose is in order to overcome the disadvantage that the anti-noise ability is weak and the edge information is lost of traditional mathematical morphology. And after setting manually several scales and several forms of structural elements in the improved algorithm, the corresponding weight coefficient of the structural elements can be determined automatically according to the information entropy. Eventually the final edge image can be got after image fusion through the series-parallel edge detector. The simulation results can be shown from two aspects of image and data: compared with the general mathematical morphology algorithm, the improved algorithm not only retains more edge information but also improves the ability of anti-noise greatly.

\section{Acknowledgements}

The authors would like to thank the editors put forward valuable opinions and suggestions which will help me improve the paper. This paper is supported by the Major National Science and Technology Programs in the "Twelfth Five-Year" Plan period (2012BAH12B03).

\section{References}

[1] Z. DjemelD and S. Tabbone, "Edge detection techniques-an overview”, International Journal of Pattern Recognition and Image Analysis, vol. 4, (1998), pp. 537-559.

[2] M. Basu, "Gaussian-based edge detection methods-a survey”, IEEE Trans Systems, Man, and Cybernetics: Part C, vol. 3, (2002), pp. 252-260.

[3] L. Yaya, L. Zhijie, Z. Haixu and W. Cunrui, "Comparison of Edge Detection Algorithm and Implementation”, Computer Engineering and Design, (2010).

[4] J. F. Canny, "A computational approach to edge detection”, IEEE Trans Pattern Analysis Machine Intelligence, vol. 6, (1986), pp. 679-698.

[5] S. Lijun, "Mathematical Morphology Research and Its Application”, Exam weekly, (2008).

[6] A. Hanbury and J. Serra, "Morphological operators on the unit circle", IEEE Transactions on Image Processing, vol. 10, no. 12, (2001), pp. 1842-1850.

[7] P. Maragos, "Differential morphology and image processing”, IEEETrans Image Processing, vol. 5, no. 6, (1996), pp. 922-937.

[8] Y. Liwen, Z. Zeng and Z. Yongji, “A gray image edge detection based on mathematical morphology method”, Foreign electronic measurement technology, (2012), 11:27 to $30+38$.

[9] E. Aptoula and S. Lefevre, “On lexicographical ordering in multi-variety mathematical morphology”, Pattern Recognition Letters, vol. 29, no. 2, (2008), pp. 109-118.

[10] W. Kun, G. Li-Qun and S. Zhen-Gang, "An edge detection algorithm based onmulti-scalemorphology”, Proceedings of the 2nd IEEE Conference on Industrial Electronics and Applications: ICIEA 2007. Washington, D C: IEEE Computer Society, (2007), pp. 2210-2213.

[11] C. Hu, C. Zhaohui and W. Shouzun, "Image Denoising Method Based on Mathematical Morphology Research”, Journal of Engineering Graphics, (2004).

[12] Z. Panhong, S. Xiaoyan, Z. Xiongwei and C. Tieyong, “A Binary Image Denoising Algorithm Based on Mathematical Morphology”, Microcomputer Information, (2010).

[13] J. Dongsheng, "Edge Detection Algorithm Based On Mathematical Morphology Research”, University of Electronic Science and Technology, (2012).

[14] R. Yan-Zhong, C. Jinghua and W. Jun, "Image Edge Detection Method Based on Mathematical Morphology and Its Application”, Journal of Henan University of Science and Technology (natural science edition), (2007).

[15] X. Guo-Bao, W. Ji, Z. Gui-Yan, Y. Yi-Xin and X. Shi-Yi, "New Algorithm Of Adaptive Edge Detection Based on Mathematical Morphology”, Journal of Computer Applications, (2009). 


\section{Authors}



Xiufang Wang received her Ph.D from Northeast Petroleum University. Now she is working in the institute of Electrical and Information Engineering and she is the department head of information engineering. Her areas of interests are mainly in the research of oil and gas signal processing.

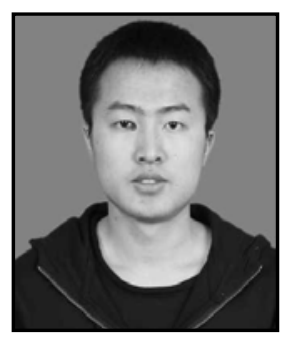

Xingyuan Zhang received his B.Eng from Shandong University of Science and Technology. Now he is a master of Northeast Petroleum University. His current areas of research interests include image processing and communication system design and development.

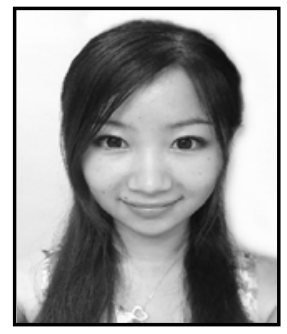

Running Gao received her B. Eng from Northeast Petroleum University and M.E.E from University of California. Once she researched in electric vehicle. Her areas of interests are mainly in Control and Robot. 
International Journal of Signal Processing, Image Processing and Pattern Recognition Vol.6, No.5 (2013) 